\title{
Enhanced Detection of Electrode Placement/Connection Errors
}

\author{
C Cooper, E Clark, PW Macfarlane \\ University of Glasgow, Glasgow, Scotland, UK
}

\begin{abstract}
Lead connection and electrode positional errors are a common problem in ECG recording. This study set out to review the sensitivity and specificity of existing criteria in the Glasgow program using an older (1997) version of the software and to produce enhancements where required for incorporation into the current version of the program still in development. 50 volunteers were recruited to the study. Arm and leg lead connection errors were introduced as were $V 1 / V 2$ and $V 2 / V 3$ connection reversals. It was shown that detection of arm lead connection errors could be enhanced from $64 \%$ to $88 \%$ at $100 \%$ specificity. Chest lead misconnections were detected with improved sensitivity. V1 and V2 reversal was much more easily detected than $V 2$ and $V 3$ reversal while maintaining high specificity.
\end{abstract}

\section{Introduction}

Despite the introduction of newer investigative techniques, electrocardiography remains one of the most widely used diagnostic tests in cardiology. Automated electrocardiography, whereby a computer program is used to interpret electrocardiograms (ECGs), was first introduced in the 1960's [1]. The University of Glasgow ECG analysis program (Glasgow Program) has been in continuous development for over 20 years [2].

Computer assisted interpretation is useful because it is quick, cheap and does not possess intra-observer variability. However, it is not without fault. Computer software is susceptible to making mistakes during interpretation of ECGs that experts would be able to interpret correctly.

Many studies have compared human ECG interpretation with computer- assisted interpretation. It has been shown that the inter-observer variability between cardiologists is equal to, if not more than the variability between computer and cardiologist [3]. The CSE study [4] has also shown that the best ECG computer programs are as good at waveform recognition as cardiologists.

It has been suggested that ECG interpretation programs could replace the cardiologist in epidemiological studies and trials [3]. In the clinical setting, however, it is recommended that all automated ECG interpretations should be interpreted by an expert over-reader to increase the accuracy of ECG interpretation.

In 1991, Willems et al [5] assessed the interpretation of ECGs by various computer programs including the Glasgow Program. The study found the sensitivity of the Glasgow Program at identifying healthy control patients, to be $94 \%$. The sensitivity of interpreting left ventricular hypertrophy and myocardial infarction was found to be $51 \%$ and $67.7 \%$ respectively. Despite continuous development of the program by enhancement of diagnostic criteria, there has been no published performance of many aspects of the Glasgow Program since the 1991 study.

\subsection{Lead misplacement}

If $1 \%$ of all recorded ECGs had an electrode connection error, then a few million ECGs each year would be wrongly recorded. Misplacement of lead connections and electrodes is known to result in the misinterpretation of electrocardiograms. In view of this, it was felt important to identify the sensitivity and specificity of lead misplacement detection by the Glasgow program and to identify ways to improve the program's performance where necessary.

Heden et al $[6,7]$ investigated lead placement errors using neural networks. In addition, they compared their results with those of the Glasgow Program available to them at the time of their study around 1994/5. They concluded that neural networks had a superior performance to more conventional criteria. However, the results suggested that there was room for improvement in respect of sensitivity of criteria for detecting arm lead reversal in the Glasgow software. These authors did not investigate misconnections involving the right leg lead.

Heden et al [7] also looked at electrode connection reversal in contiguous chest leads, e.g. V3-V4. The reported sensitivity of the Glasgow Program was extremely low. This suggested further review of the problem.

The aim of this study, therefore, was to assess 
identification of common lead connection and electrode misplacements by the Glasgow Program and if necessary, to propose potential changes in interpretation criteria in order to enhance their detection.

\section{Methods}

\subsection{Participants}

Participants comprised of 50 inpatients (mostly cardiac), staff and students at Glasgow Royal Infirmary. Informed consent was obtained from all volunteers.

\subsection{Data collection and storage}

A Burdick Eclipse 850i ECG machine was used to record a series of seven 12 lead ECGs from each participant. Participants were assigned a study number from $\mathrm{CC} 001$ to $\mathrm{CC} 050$. In addition to recording a basic 12 lead ECG with correct lead connections, an additional six ECGs were obtained with deliberate lead connection error as shown in Table 1. Each of the seven ECGs recorded was assigned a number 01 to 07 according to the configuration of the lead placements (Table 1). The participant's study number, ECG number, sex and date of birth were then entered into the ECG machine and hence the interpretation program.

Table 1. The seven different ECG lead configurations adopted to test the ability of the Glasgow Program to detect lead connection and electrode placement errors.

\begin{tabular}{|l|l|}
\hline $\begin{array}{l}\text { ECG } \\
\text { Number }\end{array}$ & Lead Configuration \\
\hline ECG 01 & Standard 12 lead ECG \\
\hline ECG 02 & $\begin{array}{l}\text { Reversal of left arm and right arm lead } \\
\text { connections }\end{array}$ \\
\hline ECG 03 & $\begin{array}{l}\text { Reversal of right arm and right leg lead } \\
\text { connections }\end{array}$ \\
\hline ECG 04 & $\begin{array}{l}\text { Reversal of left arm and right leg lead } \\
\text { connections }\end{array}$ \\
\hline ECG 05 & Reversal of V1 and V2 lead connections \\
\hline ECG 06 & Reversal of V2 and V3 lead connections \\
\hline ECG 07 & $\begin{array}{l}\text { Misplacement of V1 and V2 electrodes one } \\
\text { intercostal space too high (i.e. V1 and V2 } \\
\text { electrodes placed in the third intercostal space) }\end{array}$ \\
\hline
\end{tabular}

All ECGs were transmitted to a Siemens Megacare database to undergo interpretation by the 1997 version of the Glasgow Program. The ECG files were also extracted from the database for transfer to another development environment where they could be rerun using the latest version of the Glasgow program under development.

\subsection{Analysis}

The first $25(50 \%)$ of the ECG recordings were then used to provide a training set for developing enhancements to criteria in order to improve sensitivity of lead connection and electrode misplacement reporting. Criteria for detecting V1 and V2 interchange had to be developed as they were not incorporated into the 1997 program. Criteria to detect V1 and V2 being placed one interspace too high were not in the 1997 software but were available prior to the start of the study. A review of the interpretations helped to identify improvements to criteria. The enhanced criteria were then retested on the training set of 25 ECGs (for each lead configuration). They were also tested on 1000 abnormal ECGs from an in-house database of ECGs with a variety of abnormalities (the Glasgow 1000 ECG Database) as well as 1496 ECGs from a database of normal, adult ECGs (the Glasgow Normal Adult Database). This was to ensure that, whilst the proposed alterations in criteria might increase sensitivity, there was not any decrease in specificity.

The revised criteria were evaluated in an iterative way, and those which resulted in optimum sensitivity of detecting lead misplacement, whilst maintaining a high specificity, were selected for inclusion in the development program.

The remaining set of ECGs from the other 25 participants provided a test set by which the new criteria could be evaluated. The new criteria were also evaluated on the 1220 ECGs from the CSE database [5] because a new test set for evaluation of specificity was required. The test set was interpreted using the new criteria and the sensitivity and specificity of the interpretations were calculated. The sensitivity and specificity of the original and enhanced criteria were then compared.

\subsection{Ethical approval}

This study received ethical approval on the $23^{\text {rd }}$ of January 2008, from the Glasgow Royal Infirmary Research and Ethics Committee.

\section{Results}

\subsection{Lead misplacement}

The ages of the participants ranged from 20-84 years (average 49.42 years). There were 20 female participants and 30 male participants.

The sensitivity of lead and electrode misplacement detection by the 1997 version of the Glasgow Program is shown in Table 2. All 50 ECGs from the study population could be used for this part of the study. It was not possible to evaluate specificity of the 1997 program 
because that program was contained inside a turnkey system into which the normals data base, for example, could not be fed.

Reporting of limb lead connection misplacements (ECG 03 or ECG 04) showed the best results, namely $84 \%$ for reversal of the right arm with the right leg lead connection. ECG 05 and ECG 07 could not be evaluated by the 1997 program because criteria for the detection of these lead connection misplacements were not included in that particular release.

Final evaluation of the new criteria with the test set showed that the sensitivity of reporting all lead connection misplacements had been increased with very little subsequent decrease in the specificity (Table 2). Criteria for the detection of misplaced level of the V1 and V2 electrodes (ECG 07) were insensitive ( $0 \%$ ) but $100 \%$ specific! The sensitivity of detecting reversal of the V2 and V3 lead connections increased by over $100 \%$ but the sensitivity remained low (24\%).

Table 2. Sensitivity and specificity of the different criteria evaluated by the 1997 and development versions of the program. The ECG numbers are explained in Table 1 .

Training Set

Test set

\begin{tabular}{|c|c|c|c|c|c|}
\hline $\begin{array}{l}\text { ECG } \\
\text { No. }\end{array}$ & $\begin{array}{l}\text { Sens \% } \\
\mathrm{N}=50 \\
1997\end{array}$ & $\begin{array}{l}\text { Sens \% } \\
N=25 \\
2008\end{array}$ & $\begin{array}{l}\text { Spec \% } \\
N=2496 \\
2008\end{array}$ & $\begin{array}{l}\text { Sens \% } \\
N=25 \\
2008\end{array}$ & $\begin{array}{l}\text { Spec \% } \\
N=1220 \\
2008\end{array}$ \\
\hline 02 & 64 & 92 & 100 & 88 & 100 \\
\hline 03 & 84 & 100 & 100 & 96 & 100 \\
\hline 04 & 80 & 96 & 100 & 96 & 99 \\
\hline 05 & NA & 88 & 99.96 & 92 & 99 \\
\hline 06 & 10 & 40 & 99.88 & 24 & 99 \\
\hline 07 & $\overline{\mathrm{NA}}$ & 0 & 100 & 0 & 100 \\
\hline
\end{tabular}

\section{Discussion and conclusions}

\subsection{Lead misplacement}

The wide age range of the participants, the relatively even ratio of males to females and the varying clinical status of participants from healthy controls to cardiac inpatients permits wide application of the results of this part of the study.

In practice, reversal of the arm leads is the most common lead misplacement [6]. When not detected,

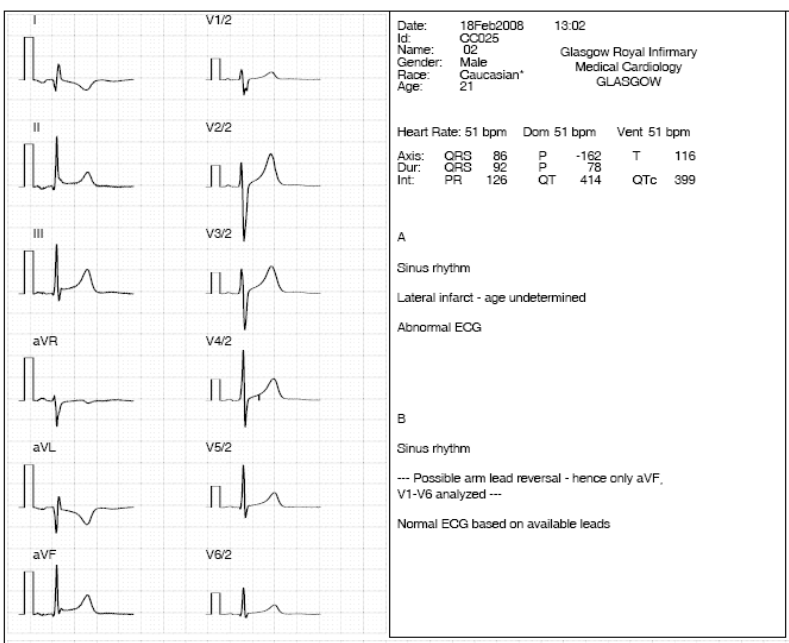

Figure 1. Automated interpretation by the 1997 version of the Glasgow Program (A) and the 2008 development program (B) of the same 12 lead ECG recorded with reversal of the arm lead connections. B is correct. The interpretations from the two reports have been amalgamated for display purposes.

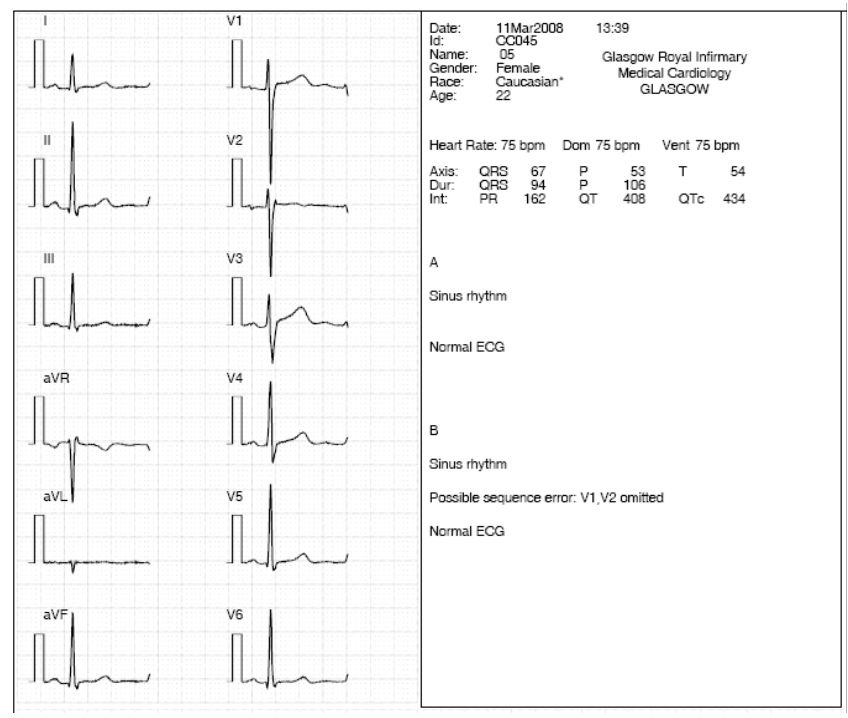

Figure 2. Automated interpretation by the 1997 version (A) of the Glasgow Program and the 2008 development program (B) of the same 12 lead ECG recorded with reversal of the V1 and $\mathrm{V} 2$ lead connections. B is correct. The interpretations from the two reports have been amalgamated for display purposes. 
reversal of the arm leads is often misinterpreted as lateral myocardial infarction (Figure 1), which has obvious clinical implications. Results identified a considerable improvement in detection of reversed arm lead connections by the development program meaning that very few such reversals will in future be reported as lateral myocardial infarction.

The study identified a need for the development of criteria to detect reversal of the V1 and V2 lead connections as these were not previously included in the program. The criteria subsequently developed allow for the identification of $92 \%$ of such misplacements to be made.

Criteria for the detection of misplacement of the V1 and V2 lead connections in the third intercostal space did not exist for the 1997 program. The criteria developed failed to detect any such misplacement. This may be because the changes in the ECG are too subtle to be detected or possibly because in the sample studied, there were no significant changes in appearances. Typically, there is an $\mathrm{rSr}^{\prime}$ complex with inverted $\mathrm{P}$ and $\mathrm{T}$ waves when this error occurs but these were not seen in this study group. It may be that such changes are encountered more frequently if the lead connections are two or more intercostal spaces too high. This was not explored in this small study. Examination of the criteria used to detect V2 and V3 lead reversal must be highly specific as the resultant changes are extremely varied. This explains why, although enhancement of criteria dramatically improved, the sensitivity of reporting V2 and V3 lead reversal, the majority of such lead misplacements will remain undetected.

This small undergraduate study has shown that it is possible to enhance the criteria for limb lead connection error. For example, arm lead reversal improved from $64 \%$ to $88 \%$ sensitivity in this study, which in turn was still superior to that obtained by Heden et al [6]. Admittedly, in the current small study, the test set did not contain any cases of atrial fibrillation. Heden et all [6] showed that conventional criteria were much less effective in this arrhythmia compared to detection of errors in patients with sinus rhythm.

On the other hand, the present study has shown a dramatic improvement in sensitivity of detecting V1-V2 interchange and a welcome improvement in sensitivity in detecting V2-V3 interchange (24\% compared to $9.3 \%$ reported in the study of Heden et al). Specificity was maintained at an extremely high level (99\%).

It was unfortunate that, in the present study, movement of electrodes one interspace higher did not produce any significant morphological changes in the ECGs, as verified by visual inspection.

\section{Conclusion}

This very small study has resulted in a modest enhancement of the criteria used to detect lead misplacement in the Glasgow program. This ensures that the next version of the program to be released will have an increased sensitivity for reporting such errors. However, there is always room for improvement and further work in this area is envisaged.

\section{Acknowledgements}

The authors would like to thank Kathryn MacLaren, Jean Watts and Shahid Latif for technical support.

\section{References}

[1] Kors JA, Van Herpen G. The Coming of Age of Computerized ECG Processing Can it Replace the Cardiologist in Epidemiological Studies and Clinical Trials? Studies in health technology and informatics 2001; 84(Pt 2):1161-1167.

[2] Macfarlane PW, Devine B, Clark E. The University of Glasgow (Uni-G) ECG Analysis Program. Computers in Cardiol 2005; 32:451-454.

[3] Shah AP, Rubin SA. Errors in the Computerized Electrocardiogram Interpretation of Cardiac Rhythm. J Electrocardiol 2007; 40:385-390.

[4] Willems JL, Arnaud P, van Bemmel JH, et al. A Reference Data Base for Multilead Electrocardiographic Computer Measurement Programs. J Am Coll Cardiol 1987; 10:13131321.

[5] Willems JL, Abreu-Lima C, Arnaud $P$ et al. The Diagnostic Performance of Computer Programs for the Interpretation of Electrocardiograms. N Engl J Med 1991; 325:1767-1773.

[6] Heden B, Ohlsson M, Edenbrandt L, Rittver R, Pahlm O and Peterson C. Artificial Neural Networks for Recognition of Electrocardiographic Lead Reversal. Am J Cardiol 1995; 75: 929-33.

[7] Heden B, Ohlsson M, HolstH, Mjoman M, Rittner R, Pahlm O, Peterson C, Edenbrandty L. Detection of frequently overlooked lead reversals using artificial neural networks. Am J Cardiol 1996; 78:600-604.

Address for correspondence

Name: $\quad$ Professor Peter Macfarlane

Full postal address: Div Cardiovascular and Medical Sciences QEB, Royal Infirmary, Glasgow G31 2ER, Scotland

E-mail address: peter.w.macfarlane@clinmed.gla.ac.uk 